\title{
ACCURACY OF FLAPLESS IMPLANT PLACEMENT WITH 3D PRINTED SURGICAL GUIDE
}

\author{
Inass Abdel Rahman AbuElmagd*, Alshaimaa Ahmed Shabaan** and Amr Salah eldin ${ }^{* * *}$
}

\begin{abstract}
Objectives: The aim of the study was to evaluate the accuracy of surgical templates for guided implant surgery using $3 \mathrm{D}$ printing.

Patients \& Methods: Twenty-four patients were examined for implant placement. Each implant site was planned virtually and a 3D printed surgical guide was constructed. The implant had been installed using the 3D printed surgical guide. Postoperative CBCT was performed, and the images were superimposed on the virtual planning images. The amount of coronal, apical, and angular deviation was calculated.
\end{abstract}

Results: A total of 48 implants were placed in 24 patients. Elven patients were completely edentulous while the other 13 patients where partially edentulous. Mean angular deviation of the implants placed in partially and completely edentulous patients $4.1 \pm 0.1$ and $3.3 \pm 0.78$ degrees respectively. While the mean deviation coronally was $1.5 \pm 0.3$ and $1.1 \pm 0.5 \mathrm{~mm}$ in partially and completely edentulous patients respectively. The deviation at the apical portion showed a mean $2.1 \pm 0.3$ and $2.2 \pm 0.5 \mathrm{~mm}$ in implants placed in partially and completely edentulous patients. Statistical significant difference between the partially and completely edentulous arches where found in coronal and angular deviation while the apical deviation showed no significant difference.

Conclusion: A high accuracy in implant placement can be achieved using 3D printed surgical guide.

KEY WORDS: Surgical Guide, Implant placement, CBCT, 3D printing, virtual planning.

\section{INTRODUCTION}

In recent years, placement of dental implants had become a more widespread method in dental clinic to restore edentulous and partially edentulous cases.
The success of dental implant was determined by the osseointegration of the implants, but, recently successful implant must also meet the restorative objectives of both function and esthetics. ${ }^{(1-4)}$

Lecturer of Oral Radiology, Faculty of Dentistry, Fayoum University

Lecturer of Oral \& Maxillofacial Surgery, Faculty of Dentistry, Fayoum University

Lecturer of Removable Prosthesis, Faculty of Dentistry, Fayoum University. 
To achieve all prosthetic objectives and avoid surgical complications, it is essential to place the implant accurately. ${ }^{(5)}$ The optimum implant position can be planned pre-operatively based on the clinical situation by making a plaster model, wax up and taking a dental $\mathrm{x}$-ray image. New technologies such as use of cone beam computerized tomography (CBCT) with 3-Dimentional construction of structures had helped in improvement of implant planning and placement. ${ }^{(6-7)}$

Several software programs for implant planning are available in the market that had been used in virtual implant placement on the 3D constructions. Those virtual planning can be transferred to the patient mouth and used to guide the implant into the optimal position. ${ }^{(7-9)}$

Different transfer techniques are described in literature. The most common method that had been used are the manually fabricated guide which produced in a dental laboratory using a system-specific drilling machine, another method is the stereolithographic templates (ST) which produced by computer-aided design/computer-aided manufacturing technology. ${ }^{(10-12)}$

The surgical guide fabrication for the radiographic planning with the previous two methods is high cost and time-consuming. New technology had been developed to transfer the planning into reality through 3D printing. This technology seems to improve surgical guide fabrication due to time and cost reduction. ${ }^{(12,13)}$

In the 3D printing surgical guide fabrication, $\mathrm{A}$ three-dimensional radiographic data set is uploaded into the planning software, which allows virtual implant planning. To transfer this planning into reality, a three-dimensional optical scan of a cast model must be uploaded into the software and superimposed to radiographically visible matching references. Based on the optical scan a virtual framework of the template can be included by mouse-click into the original planning. ${ }^{(13)}$
The sleeves can be included into the virtual frame work, and the template is then printed with any three-dimensional printer. However, though this technology seems to improve surgical guide fabrication there are no data concerning the technical accuracy of this manufacturing approach. ${ }^{(13)}$

The aim of the current study was to evaluate the accuracy of surgical templates for guided implant surgery using 3D printing.

\section{PATIENTS AND METHODS}

\section{Participants}

Twenty-four patients that require dental implant placement had participated in this study. Their age ranged from 28 to 74 years. The participants were recruited from Outpatient clinics, Faculty of Dentistry, Fayoum University.

Patient recruited in the study fulfilled the following inclusion criteria: all patients had been edentulous for more than 1 year; sufficient bone in height and width; sufficient attached mucosa around implant; had a sufficient mouth opening greater than $50 \mathrm{~mm}$ to accommodate space to the surgical guide; in a good general health. While exclusion criteria include the following: all medical condition that makes implant insertion unfavorable; need for bone grafting and / or sinus lift in the planned area; patients unlikely to able to comply with study procedures.

Completed medical and dental history was taken for all patient before the start of any dental procedure. Patients were fully informed about the treatment procedures and follow-up examinations. Informed consent was obtained from each patient prior to participation in this study.

\section{Surgical and Prosthetic planning:}

Full Arch impression were made in both arches. Casts were poured and mounted on articulator. A diagnostic wax up was made to represent the anatomy and ideal locations of the planned implants and prosthetic designs. 
Standard CBCT scanning procedures with standardized setting of $90 \mathrm{kV}, 6.3 \mathrm{~mA}$, an exposure time of $12 \mathrm{~s}$ and voxel size of $0.2 \mathrm{~mm}$ were followed for each patient. The scanning was performed by the same radiologist operating a CBCT machine (Planmeca promax 3D classic, Planmeca, Finland).

For virtual planning of implant surgical guide, the resultant CBCT were imported into the implant planning software (Blue Sky Plan ${ }^{\circledR}$ V3, Blue Sky Bio, LLC, USA). The digital image segmentation was performed and the virtual implants were placed in the most optimal position according to the surgical and prosthetic design (fig 1).

\section{Surgical Guide Printing:}

The designed virtual template was transferred as STL files and printed with a three- dimensional printing machine (Form 1+, Formlabs, USA). the metal sleeves with $5 \mathrm{~mm}$ diameter for guided surgery were manually pushed into the respective nots. (fig2)

\section{Surgical Procedures}

After injection of local anesthesia, the surgical guide was placed in the patient mouth using finger pressure to confirm proper seating of the surgical guide. The surgical guide was fixed using three equally- distributed anchor pins. (Fig3)

Drilling was performed with a $2 \mathrm{~mm}$ bur according to the metal rings of the surgical guide. Next sequential drills were used and implants were installed. The surgical guide was removed, and the cover screws were attached. (Fig 3)
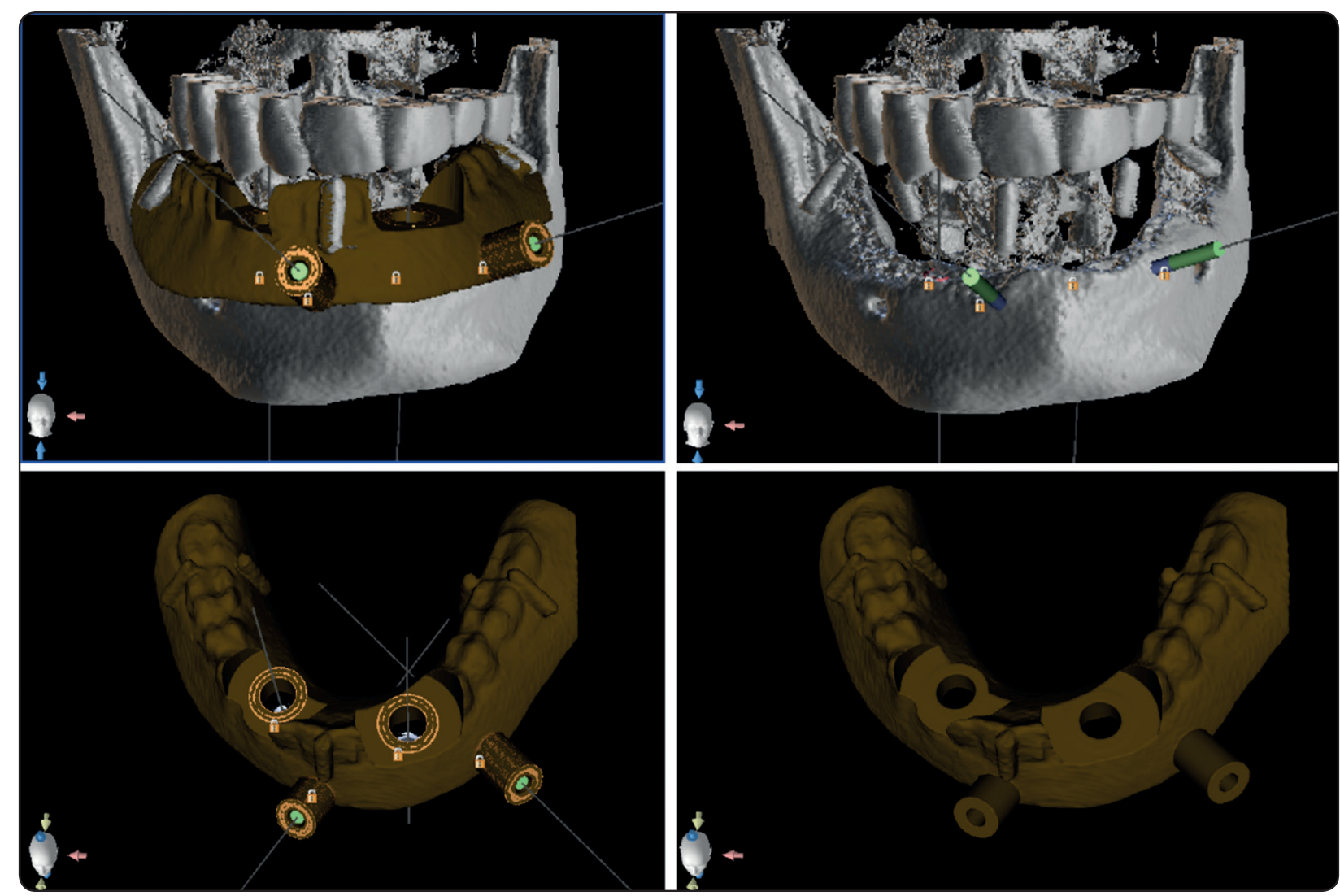

Fig. (1) Virtual deigned templet with nots for system specific metal sleeves 


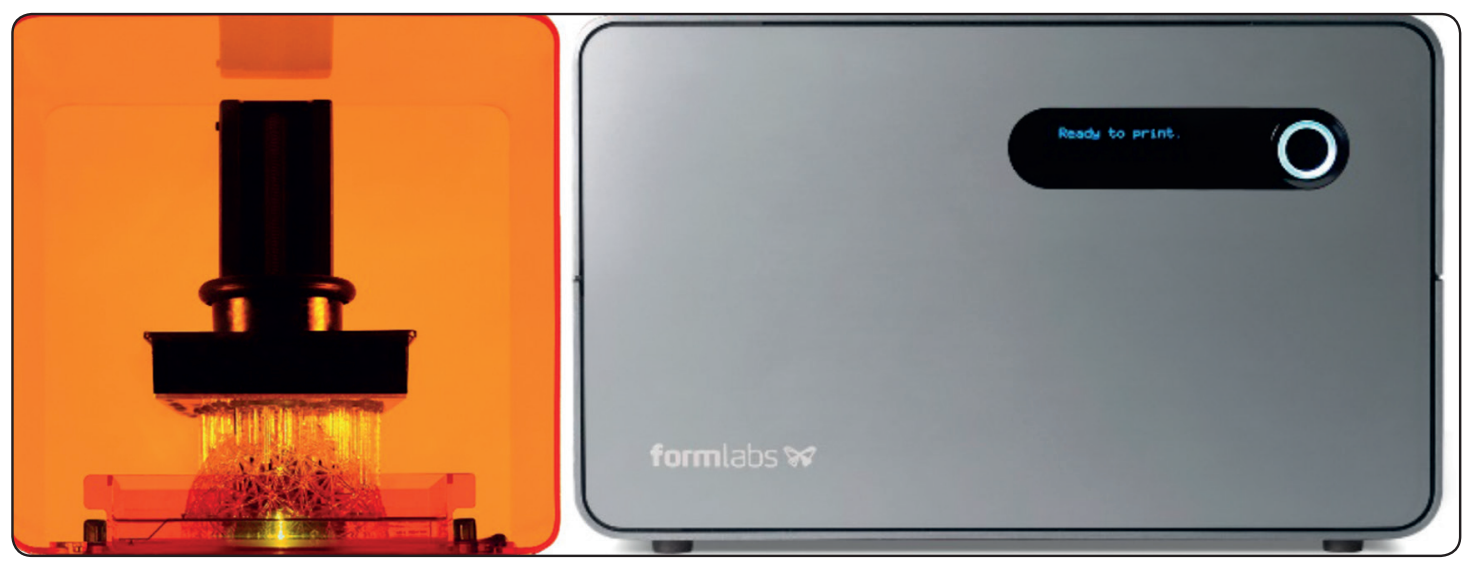

Fig. (2) The 3D printer Device

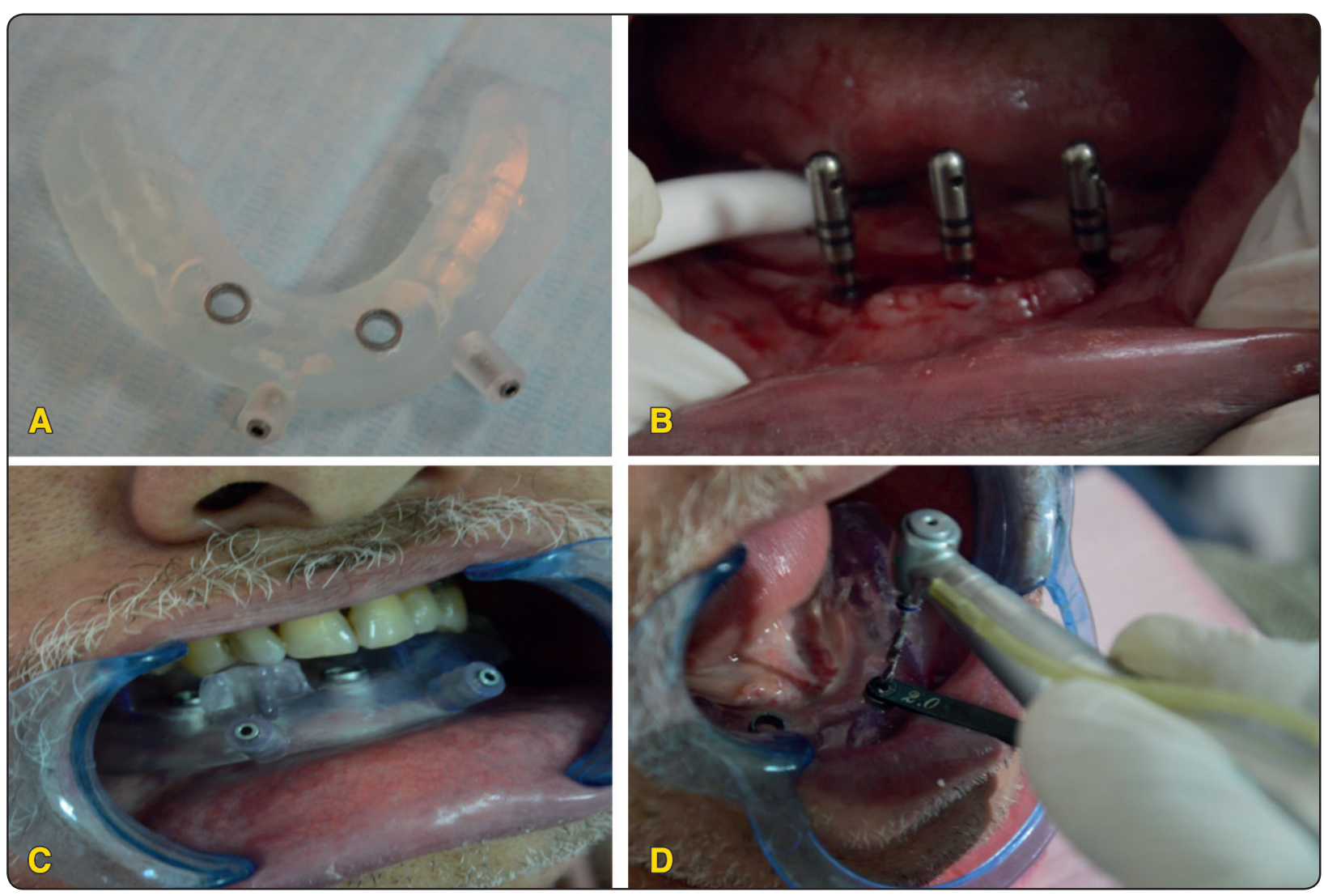

Fig. (3) Implant placement. a: Surgical guide, b: surgical guide in place, c: drilling for implant, d: implant in place. 


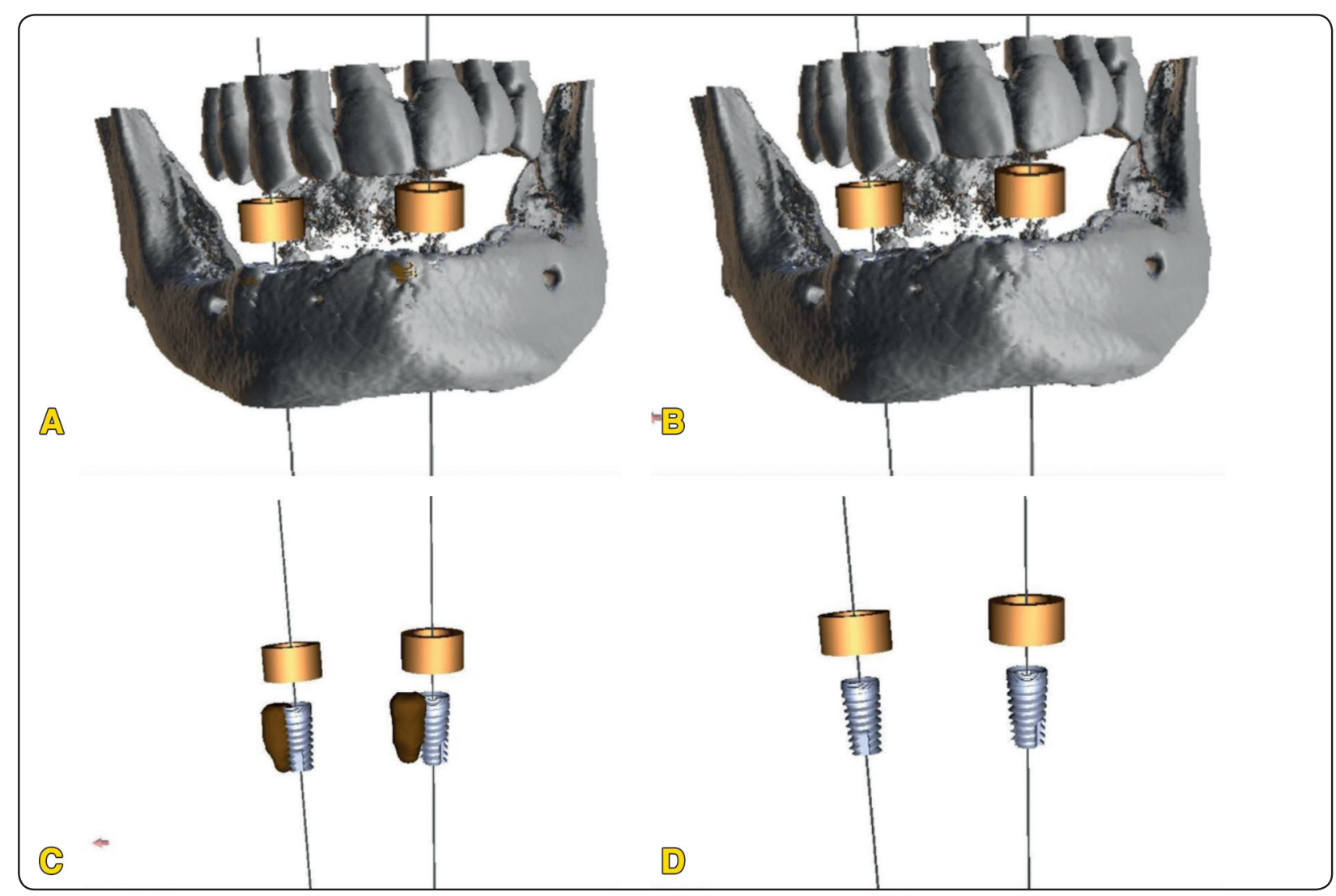

Fig. (4) Fusion of 3D images before and after Surgery.

\section{Accuracy measurement:}

For each patient, a second CBCT scan was obtained immediately after the implant placement. The software was used to fuse the first and second CBCT images of the planned and actual placed implants according to the anatomical land mark. (Fig 4)

For each planned and actually placed implant, two implant points were located in the long axes: coronal (F1) and apical (F2) locations. The distance between the centers of virtual and actually placed implant were calculated. The angle formed between the virtual and actually placed implants were also measured. (Fig 5)

All accuracy assessment was performed by the same investigator. After one week, all measurements were repeated to evaluate the reproducibility of the recording.

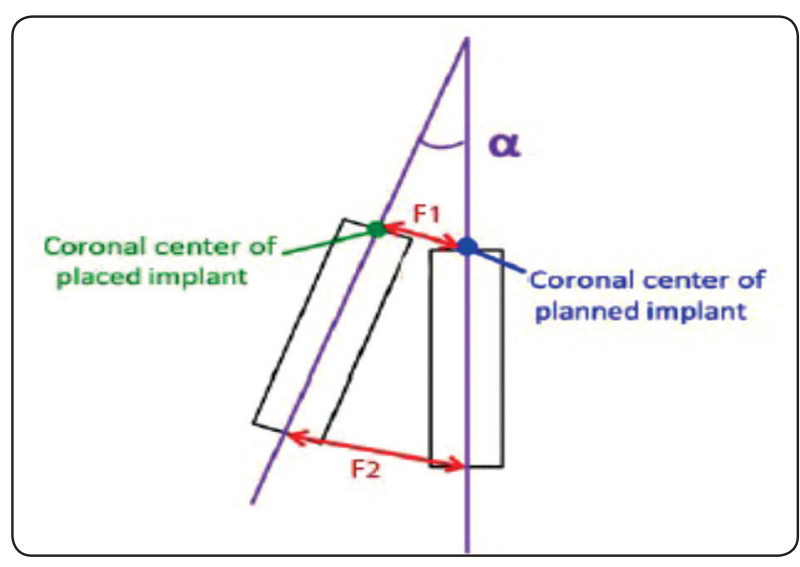

Fig. (5) Measurement of deviation between the planned implant and actually placed implants 


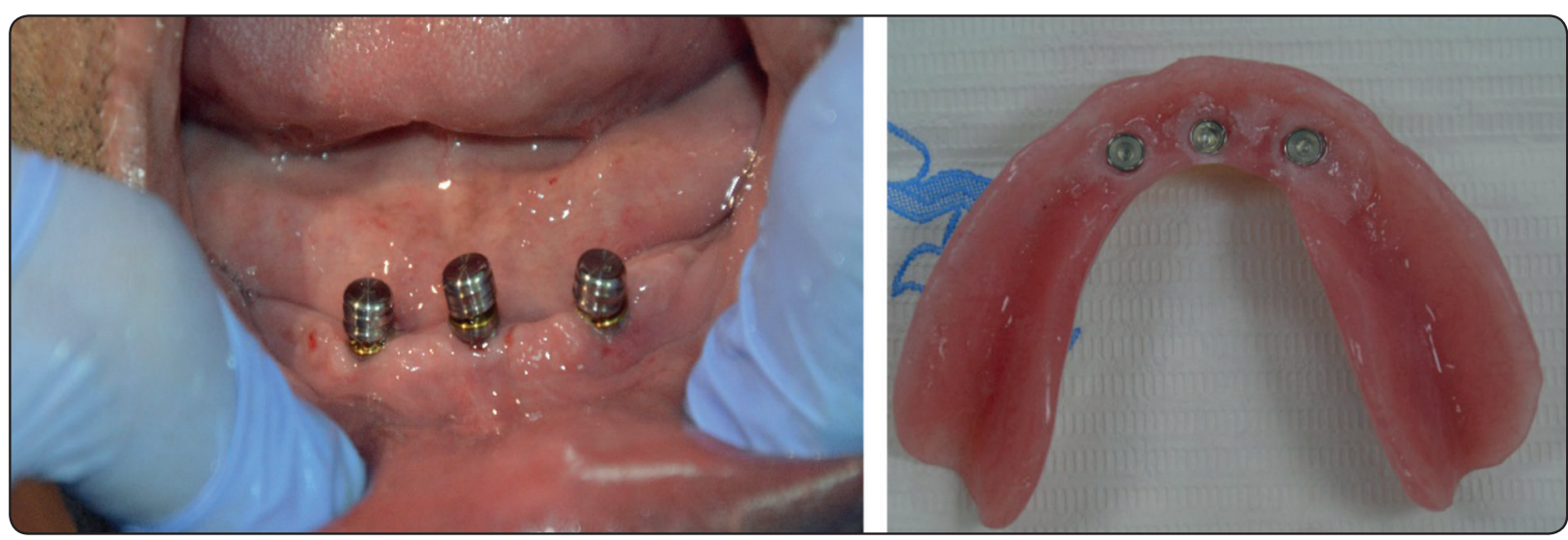

Fig. (6) The final Restoration and abutments in place.

\section{Prosthetic procedures}

The second stage surgery was done using surgical punch to expose the implant covering screw. The covering screw was removed and the healing abutment was attached. The patient was given 1 week as a healing period.

The mucosal thickness was assessed using graduated periodontal probe so that the locator abutment with a proper collar height was chosen, and then the abutment was threaded into the implant, tightened. No modification was done to the abutments after placement in either partially or completely edentulous patients (Fig 6).

\section{Statistical analysis of the data}

Data were fed to the computer and analyzed using IBM SPSS software package version 20.0. Data were fed to the computer and analyzed using IBM SPSS software package version 20.0. (Armonk, NY: IBM Corp). The Kolmogorov- Smirnov, Shapiro and D'agstino tests were used to verify the normality of distribution of variables, Comparisons between groups for categorical variables were assessed using Chi-square test (Fisher or Monte Carlo). Student t-test was used to compare two groups for normally distributed quantitative variables. Mann Whitney test was used to compare between two groups for abnormally distributed quantitative variables.
Significance of the obtained results was judged at the $5 \%$ level.

\section{RESULTS}

A total of 48 implants were placed in 24 patients. Eleven patients were completely edentulous while the other 13 patients where partially edentulous (Table1).

All implants achieved sufficient primary stability. No complications occur during implant placement. The insertion of these implants was completed manually using hand ratchet following the removal of the surgical guide.

No postoperative complications and no patients complained about the severe pain or discomfort related to implant surgery.

For the partially edentulous patients, the placed implants showed mean of $1.5 \pm 0.3 \mathrm{~mm}$ coronal deviation, $2.1 \pm 0.3 \mathrm{~mm}$ apical deviation and $3.3 \pm 0.78$ degrees angle deviation from the planned implants position.

While the completely edentulous patients, the placed implants showed mean of $1.1 \pm 0.5 \mathrm{~mm}$ coronal deviations, $2.2 \pm 0.5 \mathrm{~mm}$ apical deviation and $4.1 \pm 0.1$ degrees angle deviation from the planned implants position. 
A statistically significant difference between the partially edentulous and completely edentulous arches where found in coronal division $(\mathrm{p}<0.001)$ and angle deviation $(\mathrm{p}<0.004)$ between the planned implant and placed implant, whereas the apical deviation showed no significant difference $(\mathrm{p}=0.252)($ Table 2$)$.

All patients went on a successful fabrication and insertion of final definitive restoration with no complication or modification in the final restoration or implant's abutment angulation.

TABLE (1) Distribution of patients \& implants among the study population

\begin{tabular}{lcc}
\hline & \multicolumn{2}{c}{ State of dentition } \\
& $\begin{array}{c}\mathbf{D}^{*} \\
(\mathbf{n = 1 3})\end{array}$ & $\begin{array}{c}\mathbf{U}^{*} \\
(\mathbf{n}=\mathbf{1 1})\end{array}$ \\
\hline Sex & & \\
$\quad$ Male & $6(46.2 \%)$ & $7(63.6)$ \\
$\quad$ Female & $7(53.8 \%)$ & $4(36.4 \%)$ \\
Age & $36.2 \pm 7.2$ & $64.0 \pm 9.9$ \\
No of implants & $1(1-3)$ & $2(2-4)$ \\
1 & $8(61.5 \%)$ & $0(0 \%)$ \\
2 & $3(23.1 \%)$ & $8(72.7 \%)$ \\
3 & $2(15.4 \%)$ & $0(0 \%)$ \\
4 & $0(0 \%)$ & $3(27.3 \%)$ \\
Site & & \\
$\quad$ Maxilla & $8(61.5 \%)$ & $4(36.4 \%)$ \\
$\quad$ Mandible & $5(38.5 \%)$ & $7(63.6 \%)$ \\
\hline
\end{tabular}

*D: partially edentulous patients U: completely edentulous patients

TABLE (2) Deviation between planned and actual implant position.

\begin{tabular}{|l|c|c|c|}
\hline & $\mathrm{D}$ & $\mathrm{U}$ & $\mathrm{P}$ \\
\hline F1 (coronal) & $1.5 \pm 0.3$ & $1.1 \pm 0.5$ & $<0.001^{*}$ \\
\hline F2 (apical) & $2.1 \pm 0.3$ & $2.2 \pm 0.5$ & 0.252 \\
\hline Angle & $\begin{array}{c}3.3 \pm \\
0.78\end{array}$ & $4.1 \pm 0.1$ & $0.004^{*}$ \\
\hline
\end{tabular}

D: partially edentulous patients

$U$ : completely edentulous patients

*: Statistically significant at $p \leq 0.05$

\section{DISCUSSION}

Planning of dental implant positioning and its accurate transfer to the surgical site can be considered one of the most important factors in successful implant-supported Restoration. ${ }^{(14)}$

Placement of the implants involves risks, such as placing an implant into an anatomically unfavorable area. Prevention of risks involves precise planning using accurate radiographic methods, creating specific virtual implant placement plans and accurately transferring this information to the patients. ${ }^{(1-4)}$

Many methods and software had been used to accurately transfer the data from the planning software into the patient mouth ${ }^{(10,11)}$ There is inherent inaccuracy when accomplishing virtual surgical plans, and error can arise from the acquisition of the CBCT scans, the interpolation by software programs used for planning, the processes used to create the surgical templates, the fit and placement of the template on the patient, and operator error. A critical step is the virtual planning of the surgery and the transfer of that plan to the patient. ${ }^{(15)}$

In the current study, using of the 3D printer to the surgical guides had shown a promising potential. The printing of guides is associated with favorable cost benefits. The virtual surgical guide can be designed by the dentist/ surgeon on his own computer, thus giving the opportunity to reduce time for surgical guide fabrication and its application. The time that is needed to virtually design the guide in software take about $30 \mathrm{~min}$ per guide. Once the surgical guide had finally designed, printing is possible within minutes giving the opportunity to receive the guide within few hrs after planning.

However, printing of the surgical guide also showed disadvantages such as manual incorporation of the metal sleeves into the guide and the need of surface polishing and finishing. Moreover, the need to access printing devices that still seem too cost extensive for private ownership in dental offices. 
The results of the current study showed mean angular deviation of the implants placed in partially and completely edentulous patients $4.1 \pm 0.1$ and $3.3 \pm 0.78$ degrees respectively. These results were in agreement with the results of Naziri et al (16) who concluded median angular deviation was 4.1 degrees. That conclude us that in most cases there is no need for angled abutments when placing the implant with surgical guides. This is an advantage for the mechanical load- bearing capacity of implants and generally for long-term preservation of the peri-implant bone.

On the other hand, that results showed more angular error than found in similar investigations. Van Assche et $\mathrm{al}^{(17)}$ found a mean error of 2 degrees, Ersoyet $\mathrm{al}^{(18)}$ found a mean angular deviation of 2.6 degrees for bone-level surgical templates, and $\mathrm{Al}-$ Harbi etal ${ }^{(19)}$ found mean angular deviations of 0.7 and 0.46 degrees.

The results of deviation measurements in this study are consistent with the finding of previous studies ${ }^{(17-19)}$ with mean $1.5 \pm 0.3$ and $1.1 \pm 0.5 \mathrm{~mm}$ at the coronal portion of implants placed in partially and completely edentulous patients respectively. While the deviation at the apical portion showed a mean $2.1 \pm 0.3$ and $2.2 \pm 0.5 \mathrm{~mm}$ in implants placed in partially and completely edentulous patients. The apical deviation is considered a clinically significant because the $2 \mathrm{~mm}$ is the recommended safety margin around vital structures and could also result in significant misfit of the prosthesis. ${ }^{(4,20)}$

Vrielinck and colleagues ${ }^{(21)}$ concluded in their study that the deviation between the planned implant and actually placed implant may result from guiding tube is slightly larger than the drill diameter.

The current study showed significant difference in accuracy between in partially than in completely edentulous patients $(\mathrm{p}<0.001)$ especially in coronal part probably because of the instability of the surgical guide that results from lack of teeth as a stable point for repositioning in the completely edentulous patients. In addition to the resilience of the mucosa that affect the position of the surgical guide during the surgery and CBCT scanning.

\section{CONCLUSION}

Within the limitation of the current study, the current study indicates a very good transfer accuracy when using the 3D printed surgical guide. The technique allows surgeons to protect important anatomical structures and facilitates implant positioning in relation to intended superstructure so that the prosthetic restoration can be analyzed in advance.

\section{Competing interests}

The authors declare that they have no competing interests.

\section{Ethical considerations}

All procedures performed in the study involving human participants were in accordance with the 1964Helsinki declaration and its later amendments or comparable ethical standards. The study was approved by thein formed consent was obtained from all individual participants included in the study.

\section{REFERENCE}

1- Albrektsson T, Zarb G, Worthington P, Eriksson AR. The long-term efficacy of currently used dental implants: a review and proposed criteria of success. Int J Oral Maxillofac Implants 1986; 1:11-25.

2- Chiapasco M, Zaniboni M. Failures in jaw reconstructive surgery with autogenous onlay bone grafts for pre-implant purposes: incidence, prevention and management of complications. Oral Maxillo fac Surg Clin North Am 2011; 23:1-15.

3- Palmer RM. Risk management in clinical practice. Part 9. Dental implants. Br Dent J2010; 209:499-506.

4- Valente F, Schiroli G, Sbrenna A. Accuracy of computeraided oral implant surgery: a clinical and radiographic study. Int J Oral Maxillofac Implants 2009; 24:234-42. 
5- Attard NJ, Zarb GA. Long-term treatment outcomes in edentulous patients with implant- fixed prostheses: the Toronto study. Int J Prosthodont 2004; 17:417-24.

6- Jabero M, Sarment DP. Advanced surgical guidance technology: a review. Implant Dent 2006; 15:135-42.

7- Nickenig HJ, Eitner S. Reliability of implant placement after virtual planning of implant positions using cone beam CT data and surgical (guide) templates. J Craniomaxillofac Surg. 2007;35:207-11

8- Balshi SF, Wolfinger GJ, Balshi TJ. Guided implant placement and immediate prosthesis delivery using traditional Brånemark System abutments: a pilot study of 23 patients. Implant Dent. 2008; 17:128-35

9- Malo P, de Araujo Nobre M, Lopes A. The use of computer-guided flapless implant surgery and four implants placed in immediate function to support a fixed denture: preliminary results after a mean follow-up period of thirteen months. J Prosthet Dent. 2007 Jun;97: S26-34

10- Arisan V, Cuneyt K, Özdemir T. Accuracy of two stereolithographic guide systems for computer-aided implant placement: a computed tomography-based clinical comparative study. J Periodontol 2010; 81:43-51.

11- Jabero M, Sarment DP. Advanced surgical guidance technology: a review. Implant Dent 2006; 15:135-142.

12- Schneider D, Marquardt P, Zwahlen M, Jung RE. A systematic review on the accuracy and the clinical outcome of computer-guided template-based implant dentistry. Clin Oral Implants Res 2009; 20(Suppl 4):73-86.

13- Kühl S, Payer M, Zitzmann NU, Lambrecht JT, Filippi A. Technical accuracy of printed surgical templates for guided implant surgery with the coDiagnostiX ${ }^{\mathrm{TM}}$ software. Clin Implant Dent Relat Res. 2015;17: e177-82
14- Schmelzeisen R, Schön R, Schramm A, Gellrich NC. Computeraided procedures in implantology, distraction and craniomaxillofacial surgery. Ann R Australas Coll Dent Surg. 2002; 16:46-9

15- Cushen SE, Turkyilmaz I. Impact of operator experience on the accuracy of implant placement with stereolithographicsurgical templates: an in-vitro study. J Prosthet Dent. 2013;109:248-54.

16- Naziri E, Schramm A, Wilde F.Accuracy of computer-assisted implant placement with insertion templates. GMS Interdiscip Plast Reconstr Surg DGPW. 2016 13;5: Doc15.

17- Van Assche N, van Steenberghe D, Guerrero ME, Hirsch E, Schutyser F, Quirynen M, et al. Accuracy of implant placement based on pre-surgical planning of three-dimensional cone-beam images: a pilot study. J Clin Periodontol 2007; 34:816-21.

18- Ersoy AE, Turkyilmaz I, Ozan O, Mc-Glumphy EA. Reliability of implant placement with stereolithographic surgical guides generated from computed tomography: clinical data from 94 implants. J Periodontol 2008; 79:1339-45.

19- Al-Harbi SA, Sun AY. Implant placement accuracy when using stereolithographic template as a surgical guide: preliminary results. Implant Dent 2009; 18:46-56.

20- Juodzbalys G, Wang HL, Sabalys G. Injury of the Inferior Alveolar Nerve during Implant Placement: A Literature Review. J Oral Maxillofac Res. 2011 1;2: e1

21- Vrielinck L, Politis C, Schepers S, Pauwels M, Naert I. Image-based planning and clinical validation of zygoma and pterygoid implant placement in patients with severe bone atrophy using customized drill guides. Preliminary results from a prospective clinical follow-up study.Int J Oral Maxillofac Surg. 2003 ;32:7-14. 